\title{
Removing Weather Effects from Monochrome Images *
}

\author{
Srinivasa G. Narasimhan and Shree K. Nayar \\ Department of Computer Science, Columbia University, New York, 10027 \\ Email: \{srinivas, nayar\}@ cs.columbia.edu
}

\begin{abstract}
Images of outdoor scenes captured in bad weather suffer from poor contrast. Under bad weather conditions, the light reaching a camera is severely scattered by the atmosphere. The resulting decay in contrast varies across the scene and is exponential in the depths of scene points. Therefore, traditional space invariant image processing techniques are not sufficient to remove weather effects from images. In this paper, we present a fast physics-based method to compute scene structure and hence restore contrast of the scene from two or more images taken in bad weather. In contrast to previous techniques, our method does not require any a priori weather-specific or scene information, and is effective under a wide range of weather conditions including haze, mist, fog and other aerosols. Further, our method can be applied to gray-scale, RGB color, multi-spectral and even IR images. We also extend the technique to restore contrast of scenes with moving objects, captured using a video camera.
\end{abstract}

\section{Towards Weather-Free Vision}

Most outdoor vision applications such as surveillance, target tracking and autonomous navigation require robust detection of image features. Under bad weather conditions, however, the contrast and color of images are drastically degraded. Hence, it is imperative to remove weather effects from images in order to make vision systems more reliable. Unfortunately, the effects of bad weather increase exponentially with the distances of scene points from the sensor. As a result, conventional space invariant filtering techniques fail to adequately remove weather effects from images.

Recently, there has been an increased interest in the image processing and vision communities on issues related to imaging under bad weather. Kopeika et al $[5,17]$ de-blur atmospherically degraded images using a weather-predicted atmospheric modulation transfer function, and an a-priori estimate of the distance from which the scene is imaged. Oakley et al $[12,15]$ describe a physics based method to restore scene contrast without using predicted weather information. However, they assume that scene depths are known beforehand, and approximate the distribution of radiances in the scene by a single gaussian with known variance.

Narasimhan and Nayar [10] analyze the color variations in the scene under different weather conditions based on the dichromatic atmospheric scattering model proposed in

${ }^{*}$ This work was supported in parts by a DARPA Human ID Contract (N00014-00-1-0916) and an NSF Award (IIS-99-87979).
[11]. Using constraints on scene color changes, they compute complete 3D structure and recover clear day scene colors from two or more bad weather images. However, they assume that the atmospheric scattering properties do not change with the wavelength of light. This property holds over the visible spectrum only for certain weather conditions such as fog and dense haze. Furthermore, the dichromatic model is ambiguous for scene points whose colors match the color of fog or haze.

Polarizing filters have been used widely by photographers to reduce haziness in images. However, polarization filtering alone does not ensure complete removal of haze. Schechner et al [13] further analyzed 2 or more polarization filtered images to compute scene structure and dehaze images. Another work by Grewe and Brooks [2] uses wavelet based fusion of multiple bad weather images to get a less blurred image.

In this paper, we present a physics based method to restore contrast completely from two or more images taken in bad weather. A monochrome atmospheric scattering model that describes how scene intensities are effected by weather conditions is presented. This model is valid in both the visible and near-IR spectra, and for a wide range of weather conditions such as mist, haze, fog and other aerosols. Based on this model, an automatic algorithm to recover complete scene structure from two images taken under different weather conditions is presented. Using the computed structure, contrast is restored from a single image of the scene. We extend our algorithms to handle video and describe a simple heuristic to restore contrasts of moving objects in the scene whose depths are unknown.

The entire analysis in this paper is done for monochrome (narrow spectral band) images. However, we show that our methods can be applied to images taken using gray-scale, wide-band RGB, multi-spectral and also narrow-band IR cameras. The effectiveness of these sensors under various weather conditions is discussed.

\section{Atmospheric Scattering Models}

Scattering of light by physical media has been one of the main topics of research in the atmospheric optics and astronomy communities. In general, the exact nature of scattering is highly complex and depends on the types, orientations, sizes and distributions of particles constituting the media, as well as wavelengths, polarization states and directions of the incident light $[1,3]$. Here, we focus on two models - attenuation and airlight, that form the basis of our work. 


\subsection{Attenuation and Airlight}

The attenuation model describes the way light gets attenuated as it traverses from a scene point to the observer. The attenuated irradiance is given by (see [7, 10]),

$$
E_{d t}(d, \lambda)=\frac{E_{\infty}(\lambda) r(\lambda) e^{-\beta(\lambda) d}}{d^{2}} .
$$

where, $d$ is the depth of the scene point from the observer and $\lambda$ is the wavelength. $\beta(\lambda)$ is called the scattering coefficient of the atmosphere; it represents the ability of a unit volume of atmosphere to scatter light in all directions. $\beta(\lambda) d$ is called the optical depth of the scene point. $E_{\infty}$ is the horizon brightness and $r$ is a function that describes the reflectance properties and the sky aperture ${ }^{1}$ of the scene point.

The second atmospheric scattering model we consider is called the airlight model. The airlight model quantifies how a column of atmosphere acts as a light source by reflecting environmental illumination towards an observer. The irradiance due to airlight is given by (see [6]),

$$
E_{a}(d, \lambda)=E_{\infty}(\lambda)\left(1-e^{-\beta(\lambda) d}\right) .
$$

The total irradiance $E$ received by the sensor is the sum of irradiances due to attenuation and airlight respectively :

$$
E(d, \lambda)=E_{d t}(d, \lambda)+E_{a}(d, \lambda) .
$$

\subsection{Wavelength Dependence of Scattering}

Generally different wavelengths of light are scattered differently by atmospheric particles. Interesting atmospheric phenomena such as the blueness of the sky and the bluish haze of distant mountains are examples of the wavelength selective behavior of atmospheric scattering $[4,8]$. In these cases, the blue wavelengths are scattered more compared to other visible wavelengths. On the other hand, fog and dense haze scatter all visible wavelengths more or less the same way.

Over the visible spectrum, Rayleigh's law of atmospheric scattering provides the relationship between the scattering coefficient $\beta$ and the wavelength $\lambda[6]$ :

$$
\beta(\lambda) \propto \frac{1}{\lambda^{\gamma}},
$$

where, $0 \leq \gamma \leq 4$ depending on the exact particle size distribution in the atmosphere. For pure air, $\gamma=4$; short (blue) wavelengths dominate and we see the clear blue sky. For fog, $\gamma \approx 0$; all wavelengths are scattered equally and we see grayish (or white) fog. A wide gamut of atmospheric conditions arise from aerosols whose particle sizes range between minute air molecules $\left(10^{-4} \mu \mathrm{m}\right)$ and large fog droplets $(1-10 \mu m)$. Such aerosols (eg., mild haze and mist) show a significant wavelength selectivity $(0<\gamma<4)$.

\footnotetext{
${ }^{1}$ Solid angle subtended by the area of sky visible to a scene point.
}

\subsection{Weather Conditions and Camera Response}

Different cameras measure irradiance over different color bands. Some examples include, gray-scale cameras (entire visible spectrum), conventional color cameras (3 broad bands R, G and B), and multi-spectral cameras (multiple narrow color bands). In the appendix, we derive an expression for the brightness recorded by a monochrome (narrow spectral band) camera, using (3). In this derivation, we assume that the scattering coefficient $\beta$ remains constant within the spectral bandwidth of the monochrome camera.

Keeping the above assumption in mind, we now discuss under what weather conditions can our methods be applied to various sensors. Recall from section 2.2 that the scattering coefficient for fog and dense haze remains more or less constant over the visible spectrum. Accordingly, a broad band RGB or gray-scale camera suffices to analyze images taken in fog and dense haze. For other aerosols such as mild haze and mist, multi-spectral cameras or cameras fitted with narrow band filters should be used in order to apply our methods. Finally, scattering coefficients of most weather conditions vary significantly in the near-IR spectrum [16] and hence, narrow-band IR cameras have to be used for the analysis beyond the visible wavelengths.

\section{Contrast Restoration of Iso-Depth Regions}

We now describe a simple method to restore scene contrast from one bad weather image, using depth segmentation of the scene. We define depth segmentation as the extraction of iso-depth regions in the scene. Note this does not mean that actual scene depths have to be known. In several situations, it may be easy to interactively provide the necessary segmentation. For instance, in urban scenes with frontal views of buildings, a user can easily mark out regions that roughly have the same depths. Later, we will present two automatic depth segmentation techniques using images taken under different weather conditions.

Consider an image taken in bad weather. The brightness at any pixel recorded by a monochrome camera is given by,

$$
E=I_{\infty} \rho e^{-\beta d}+I_{\infty}\left(1-e^{-\beta d}\right)
$$

where, $I_{\infty}$ is termed as sky intensity (see appendix). We call $\rho$ the normalized radiance of a scene point; it is a function of the scene point reflectance (BRDF), normalized sky illumination spectrum, and the spectral response of the camera, but not the weather condition defined by $\left(\beta, I_{\infty}\right)$.

Now consider two scene points $P_{i}$ and $P_{j}$ at the same depth $d$ from a sensor. Their pixel intensities are given by,

$$
\begin{aligned}
& E^{(i)}=I_{\infty} \rho^{(i)} e^{-\beta d}+I_{\infty}\left(1-e^{-\beta d}\right), \\
& E^{(j)}=I_{\infty} \rho^{(j)} e^{-\beta d}+I_{\infty}\left(1-e^{-\beta d}\right) .
\end{aligned}
$$


The observed contrast between $P_{i}$ and $P_{j}$ can be defined as,

$$
\frac{E^{(i)}-E^{(j)}}{E^{(i)}+E^{(j)}}=\frac{\rho^{(i)}-\rho^{(j)}}{\rho^{(i)}+\rho^{(j)}+2\left(e^{\beta d}-1\right)} .
$$

This shows that the contrast degrades exponentially with the depths of scene points in bad weather. Eliminating the unknown $e^{-\beta d}$ from (6), we obtain,

$$
\frac{1-\rho^{(i)}}{1-\rho^{(j)}}=\frac{I_{\infty}-E^{(i)}}{I_{\infty}-E^{(j)}}
$$

For robustness, we consider all the pixels at the same depth,

$$
\frac{1-\rho^{(i)}}{\sum_{j}\left(1-\rho^{(j)}\right)}=\frac{I_{\infty}-E^{(i)}}{\sum_{j}\left(I_{\infty}-E^{(j)}\right)} .
$$

Then, the normalized radiance of any scene point is obtained using,

$$
\rho^{(i)}=1-\left(\sum_{j} 1-\sum_{j} \rho^{(j)}\right) \frac{I_{\infty}-E^{(i)}}{\sum_{j}\left(I_{\infty}-E^{(j)}\right)} .
$$

This procedure is repeated independently for each depth in the scene. So, if we have a priori depth segmentation of the scene and have measured the sky intensity $I_{\infty}$, then $\rho^{(i)}$ can be computed up to a linear factor $\sum_{j} \rho^{(j)}$. Since $\rho$ is independent of the weather condition, we have restored the contrast of the scene using just one bad weather image.

\section{Depth Edges from Two Weather Conditions}

In this section, we present a simple cue to automatically locate the depth edges (discontinuities) present in a scene from two monochrome images taken under different but unknown weather conditions. Note that closed contours of depth edges can be used for depth segmentation. In outdoor surveillance applications, video cameras capture the same scene (albeit with moving objects) over long periods of time during which the weather may change. Also, depth edges in the static portion of any scene have to be computed just once and not for every video frame. Hence, we see this as an initialization step that needs to be done before applying the contrast restoration algorithm of section 3 to all frames.

Consider a small image neighborhood that corresponds to scene points that are at the same depth from an observer (i.e., no depth edges present). We call such a neighborhood as an iso-depth neighborhood. From (5), the average brightness of an iso-depth neighborhood is,

$$
\bar{E}=\left[I_{\infty} e^{-\beta d}\right] \bar{\rho}+\left[I_{\infty}\left(1-e^{-\beta d}\right)\right],
$$

and the standard deviation of the neighborhood is,

$$
\sigma_{E}=\sqrt{\frac{1}{n} \sum_{i=1}^{n}\left(E^{(i)}-\bar{E}\right)^{2}} .
$$

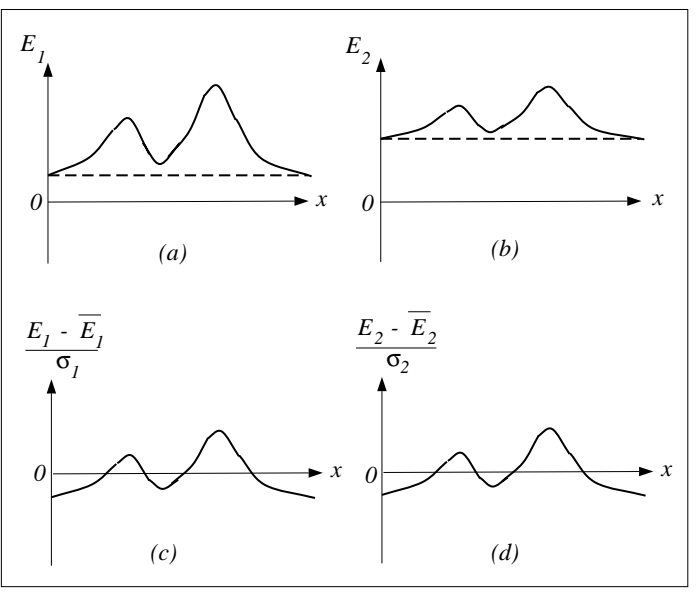

Figure 1: Invariance of iso-depth neighborhoods to weather conditions. $(a)-(b)$ Signals representing the intensities of a neighborhood of iso-depth scene points in two weather conditions. Airlight (dashed lines) is constant for the entire neighborhood. $(c)-(d)$ Normalized signals in the two weather conditions match exactly.

Using (5), we simplify to obtain,

$$
\sigma_{E}=I_{\infty} e^{-\beta d} \sqrt{\frac{1}{n} \sum_{i=1}^{n}\left(\rho^{(i)}-\bar{\rho}\right)^{2}} .
$$

Normalizing the pixel values in the neighborhood, we get,

$$
\frac{E^{(i)}-\bar{E}}{\sigma_{E}}=\frac{\left(\rho^{(i)}-\bar{\rho}\right)}{\sqrt{\frac{1}{n} \sum_{i=1}^{n}\left(\rho^{(i)}-\bar{\rho}\right)^{2}}} .
$$

For iso-depth neighborhoods, clearly the above equation is invariant to the weather condition $\left(\beta, I_{\infty}\right)$. More importantly, the invariance does not hold for a neighborhood that contains depth edges. This is easily explained as follows. The airlight does not remain constant across a neighborhood with depth discontinuities. Hence, subtracting the mean (as in (14)) will not remove the airlight completely.

Now let us consider two images captured under different weather conditions. We assume that the two images are taken under similar daylight distributions. However, the magnitudes of the distributions $\left(I_{\infty}\right)$ may vary. Figures 1(a) and (b) illustrate the brightnesses within an iso-depth neighborhood under two weather conditions. Figures 1(c) and (d) show that the normalized signals under the two weather conditions match perfectly. On the other hand, figure 2 illustrates that normalized signals of scene neighborhoods that contain depth edges, do not match. Normalized SSD can be used to determine the quality of the match.

It is interesting to note what happens if we treat the entire image as a single neighborhood. Applying normalized SSD to two images of a scene, a poor match implies that the weather condition changed between the two images, and a 


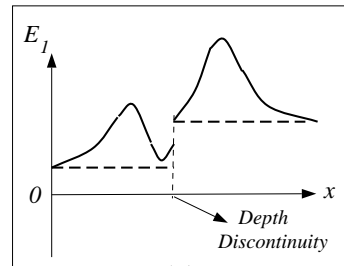

(a)

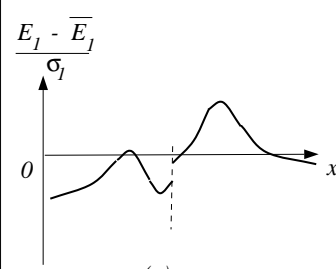

(c)

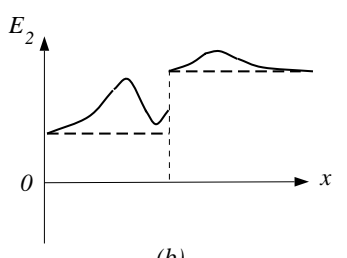

(b)

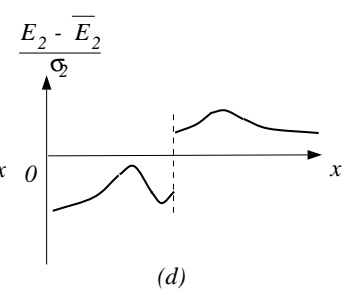

Figure 2: Illustration of scene intensities of a neighborhood that has a depth edge. $(a)-(b)$ Signals representing the intensities of the neighborhood under two weather conditions. Airlight (dashed lines) varies across the neighborhood. $(c)-(d)$ Normalized signals in the two weather conditions do not match.

good match implies otherwise. For this, the scene should have at least two different depths and the images should be linearized using the radiometric response function of the camera. This cue is helpful in deciding which frames can be used to compute depth edges in a video sequence.

Figure 3 shows the experimental results of classifying image edges into reflectance edges and depth edges for a real scene captured under two different foggy conditions. The time between the capture of the images was about half an hour. The edge map of one of the images was computed using the Canny edge detector. For each edge pixel, we considered $15 \times 15$ neighborhoods around the pixel in the two images. We applied normalized SSD to match these neighborhoods. For the depth edges, the normalized SSD value was high; for the reflectance edges, the value was low. The depth edges are shown in white and reflectance edges are shown in black (figure 3(d)). Note if both reflectance edges and depth edges are within the same neighborhood, this method may misclassify the reflectance edges as depth edges.

\section{Scene Structure}

In the previous section, we described a method to locate depth discontinuities from two bad weather images. Note, however, that normalized SSD is effective only in textured neighborhoods (reflectance edges and depth discontinuities). In other words, normalized SSD is not reliable for "flat" intensity regions and regions where depth changes are gradual. Moreover, due to the blurring seen in images taken under poor visibility conditions, the edge maps may not be reliable enough to create closed contours of depth discontinuities (needed for depth segmentation).

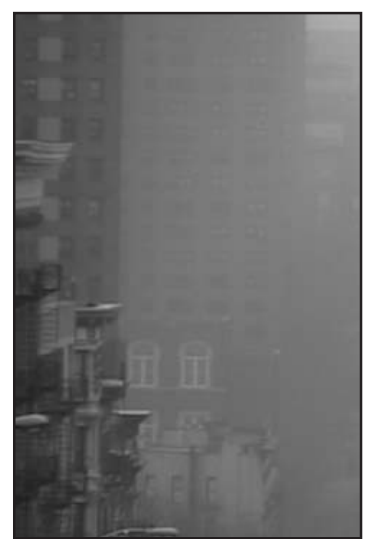

(a)

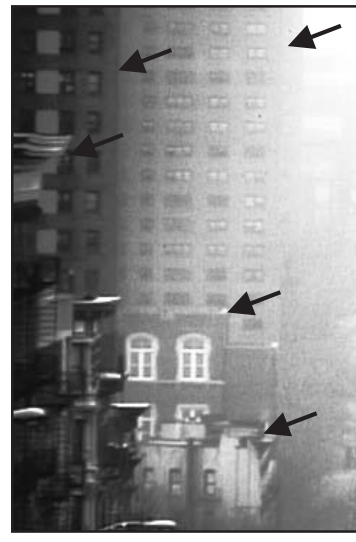

(c)

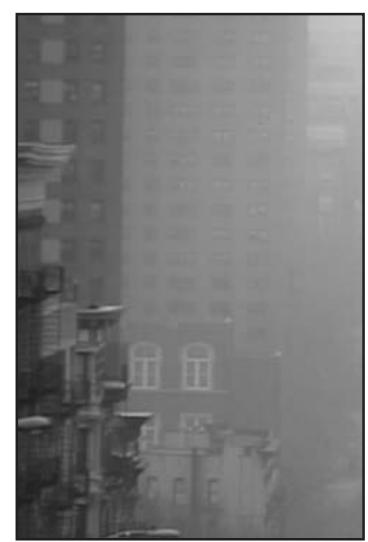

(b)

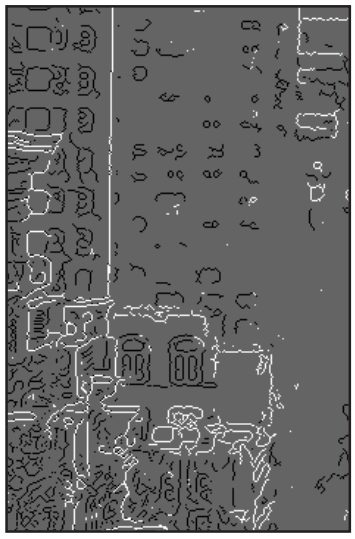

(d)
Figure 3: Classification of images edges into reflectance edges and depth edges. $(a)-(b)$ Images of the same scene captured under different fog conditions (half an hour apart). (c) The image in $(a)$ is histogram equalized to aid visualization of depth edges (shown using arrows $).(d)$ White pixels denote depth edges and black pixels denote reflectance edges. Note that the edge detector was applied to the original image in $(a)$ and not the histogram equalized image.

In this section, we present a method to compute complete structure of an arbitrary scene, from two images taken under different weather conditions. In contrast to the methods proposed in $[11,10]$ that require color images ( 3 color channels), our algorithm can be applied to both gray-scale as well as color images.

Consider the observed pixel values $E_{1}$ and $E_{2}$ of a scene point under two weather conditions $\left(\beta_{1}, I_{\infty_{1}}\right)$ and $\left(\beta_{2}, I_{\infty_{2}}\right)$. Let us examine how the brightness of this scene point changes from the first weather condition to the second. From (5):

$$
\begin{gathered}
E_{1}=I_{\infty_{1}} \rho e^{-\beta_{1} d}+I_{\infty_{1}}\left(1-e^{-\beta_{1} d}\right) \\
E_{2}=I_{\infty_{2}} \rho e^{-\beta_{2} d}+I_{\infty_{2}}\left(1-e^{-\beta_{2} d}\right)
\end{gathered}
$$




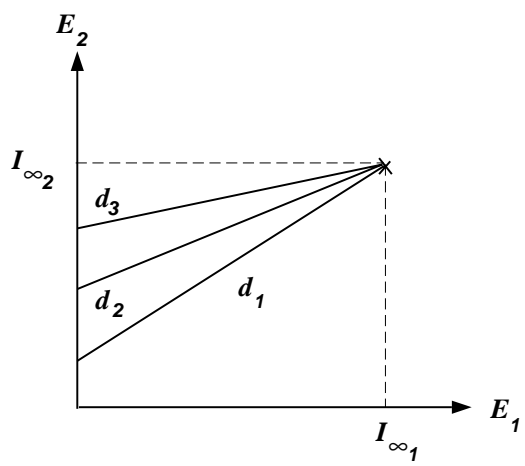

Figure 4: Plot of the pixel values $E_{1}$ observed under one weather condition versus the corresponding pixel values $E_{2}$ observed under another weather condition. Each line represents all the scene points at the same depth from the sensor. All iso-depth lines intersect at the horizon brightnesses $\left(I_{\infty_{1}}, I_{\infty_{2}}\right)$ of the two weather conditions.

Eliminating $\rho$ from (15) we get,

$$
E_{2}=\left[\frac{I_{\infty_{2}}}{I_{\infty_{1}}} e^{-\left(\beta_{2}-\beta_{1}\right) d}\right] E_{1}+\left[I_{\infty_{2}}\left(1-e^{-\left(\beta_{2}-\beta_{1}\right) d}\right)\right]
$$

which is linear in $E_{1}$ and $E_{2}$. Also, for the two weather conditions, the coefficients of the linear equation depend only on scene depth. In other words, for iso-depth scene points, the plot of $E_{1}$ versus $E_{2}$ is a straight line.

Interestingly, if we substitute $E_{1}=I_{\infty_{1}}$ in (16), we get $E_{2}=I_{\infty_{2}}$, irrespective of the depth $d$. Therefore, the point $\left(I_{\infty_{2}}, I_{\infty_{1}}\right)$ lies on the straight lines corresponding to different depths in the scene (see figure 4). In other words, the intersection of straight lines corresponding to different depths yields the sky intensities $I_{\infty_{1}}$ and $I_{\infty_{2}}$. To compute sky intensities quickly, we divide the two images into blocks and within each block we fit lines to the $\left(E_{2}, E_{1}\right)$ pairs of scene points. If the fit is good, we decide that the scene points in the block are at the same depth. Then, we use at least two such iso-depth blocks to estimate sky intensities.

Substituting the values of $I_{\infty_{1}}$ and $I_{\infty_{2}}$ in (16), we obtain the scaled depth of each scene point:

$$
\left(\beta_{2}-\beta_{1}\right) d=-\ln \frac{I_{\infty_{2}}-E_{2}}{I_{\infty_{1}}-E_{1}}-\ln \frac{I_{\infty_{1}}}{I_{\infty_{2}}} .
$$

Thus, we have computed the depth map of a scene from two images taken under different weather conditions.

\section{Contrast Restoration using Scene Structure}

In section 3, we described a method to restore scene contrast given a depth segmentation of the scene. This method is simple and effective for scenes where depth changes are abrupt (for example, an urban scene with frontal views of buildings). However, it is hard to define good depth segmentation when scene depths change gradually (for instance, a natural scene with mountains or an urban scene with a oblique view of a road). In this section, we present a method to restore contrast of an arbitrary scene using scaled depths (17) of scene points.

We assume that there exists a black patch ${ }^{2} E_{\text {black }}$ in the scene whose radiance is zero. For instance, window interiors in urban scenes are dark and can be assumed to be black. We can either mark such a patch manually or detect one automatically from the image (see [9]). Since the apparent brightness of the black patch is solely due to airlight, its optical depth can be computed as,

$$
\beta d_{\text {black }}=-\ln \left(1-E_{\text {black }} / I_{\infty}\right) .
$$

Then, the optical depth of any other scene point $P_{i}$ is obtained using,

$$
\beta d_{i}=\left(\beta d_{\text {black }}\right)\left(\frac{d_{i}}{d_{\text {black }}}\right)
$$

where, the second term can be computed using the ratio of scaled depths (see (17)). Then, the normalized radiance $\rho_{i}$ of the scene point $P_{i}$ is estimated using (5). Recall that $\rho$ does not depend on the weather condition $\left(\beta, I_{\infty}\right)$. Thus, by computing $\rho$ for each scene point, we restore contrast of the entire scene.

Note that structure computation requires two images to be taken under different weather conditions but under similar daylight spectra. However, contrast can be restored from a single image of the scene taken under arbitrary weather and illumination conditions. Figure 5(a) shows experiments with a synthetic scene consisting of a stack of cylinders with random brightness values. To this image, two different amounts of fog are added according to the model described in (5). Figure 5(c) and (d) shows the results of applying the structure computation and contrast restoration algorithms to images of the synthetic scene.

\section{Experiments with Video : Moving Objects}

Consider an outdoor surveillance video camera capturing a scene (with moving objects) over an extended period of time. We would like to process this video in real-time to obtain a weather-free video. For the purposes of discussion, we define the static part of the scene as the background, and the moving objects in the scene as the foreground. The foreground objects can be separated from the background using any background subtraction method (for instance, [14]). Then, weather-free video is obtained using an algorithm that has the following two stages:

- Initialization stage : We first detect any change in weather condition using normalized SSD (section 4). Then, the two frames that correspond to the different

\footnotetext{
${ }^{2}$ The black scene patch will not appear black in the image due to the addition of airlight.
} 

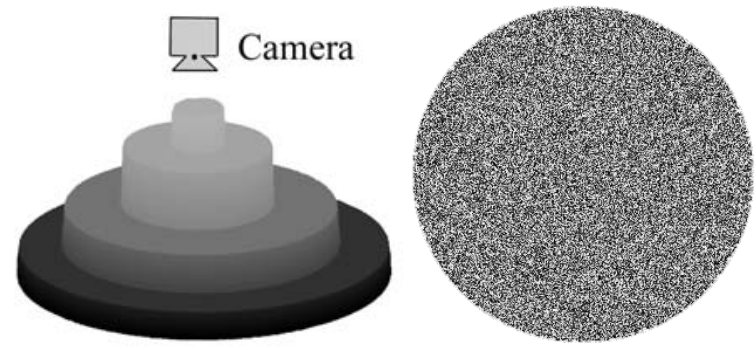

(a)
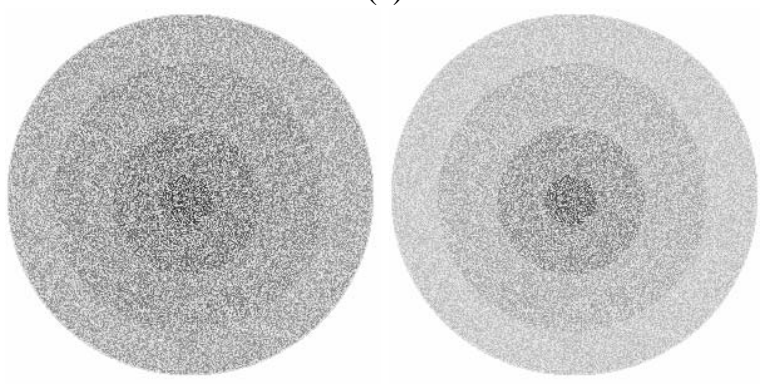

(b)

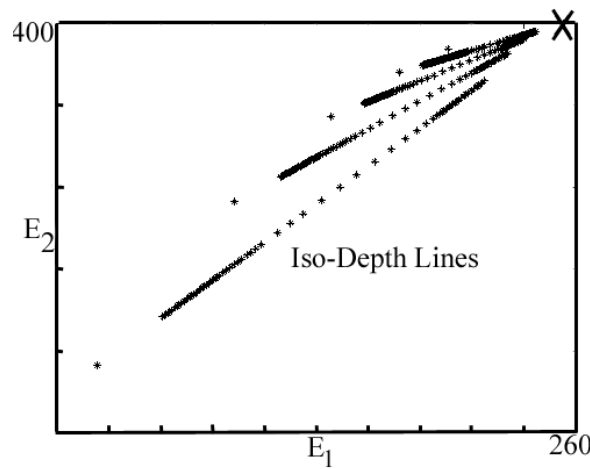

(c)

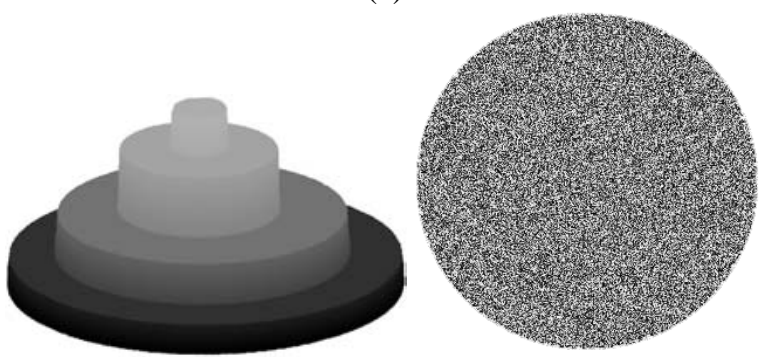

(d)

Figure 5: Experiments on a synthetic scene - a stack of discs textured with random gray dots. (a) On the left is the $3 \mathrm{D}$ structure and on the right is an image of the top view of the scene. The gray levels on the structure are used only to illustrate the disks better. (b) Two different amounts of fog are added to the image in (a). (c) Iso-depth lines shown in the plot of pixel values under the first weather condition versus the corresponding pixel values under the second weather condition. $X$ mark shows the intersection $\left(I_{\infty_{2}}, I_{\infty_{1}}\right)$ of all the iso-depth lines. $(d)$ The recovered structure and contrast restored image. weather conditions are used to compute scaled depths of the background scene (section 5).

- Contrast Restoration : Note that the methods we described hitherto cannot be used to restore contrast of moving objects since their depths are unknown. Therefore, heuristics are needed to assign depths to foreground objects. One conservative heuristic is to examine the depths in a neighborhood around each moving object and assign the minimum depth to it. The algorithm presented in section 6 can then applied to the entire frame to restore scene contrast. We have yet to study such heuristics in detail.

Experimental results with a video of a traffic scene taken under foggy conditions are shown in figure 6 . We used an offthe-shelf 8-bit digital video camera and captured two short video clips half an hour apart. We averaged 100 frames in each video clip to reduce noise and used the resulting images to compute structure of the background scene (buildings). The scaled depths in the road region were linearly interpolated. Then, contrasts of buildings, the road and moving vehicles were restored for each frame of the video. Notice the significant increase in contrast at various depths in the scene (figures 6(d-e)). In our initial implementation, contrast restoration was applied to the video off-line. Current work includes creating a real-time "de-weathering system".

\section{Summary}

In this paper, we addressed the problem of restoring the contrast of atmospherically degraded images and video. We presented methods to locate depth discontinuities and to compute structure of a scene, from two images captured under different weather conditions. Using either depth segmentation (regions within closed contours of depth edges) or scene structure (scaled depths), we then showed how to restore contrast from any image of the scene taken in bad weather. Note although structure computation requires changes in weather, the contrast restoration algorithms do not. The entire analysis is presented for monochrome images, however our methods can be applied to images captured using multispectral cameras, IR cameras and the usual broadband RGB and gray-scale cameras.

\section{References}

[1] S. Chandrasekhar. Radiative Transfer. Dover Publications, Inc., 1960.

[2] L. L. Grewe and R. R. Brooks. Atmospheric attenuation reduction through multisensor fusion. Sensor Fusion: Architectures, Algorithms, and Applications II, Proceedings of SPIE, 3376, April 1998.

[3] Van De Hulst. Light Scattering by small Particles. John Wiley and Sons, 1957.

[4] N.S. Kopeika. General wavelength dependence of imaging through the atmosphere. Applied Optics, 20(9), May 1981. 


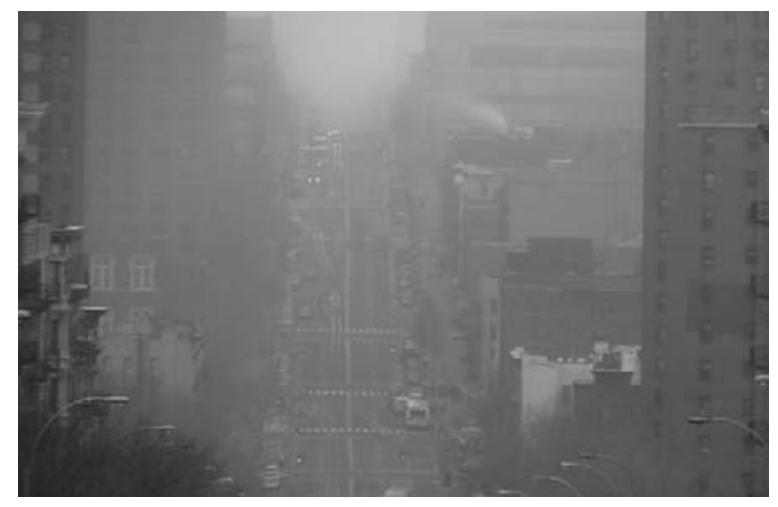

(a) Scene imaged at 5:00pm.

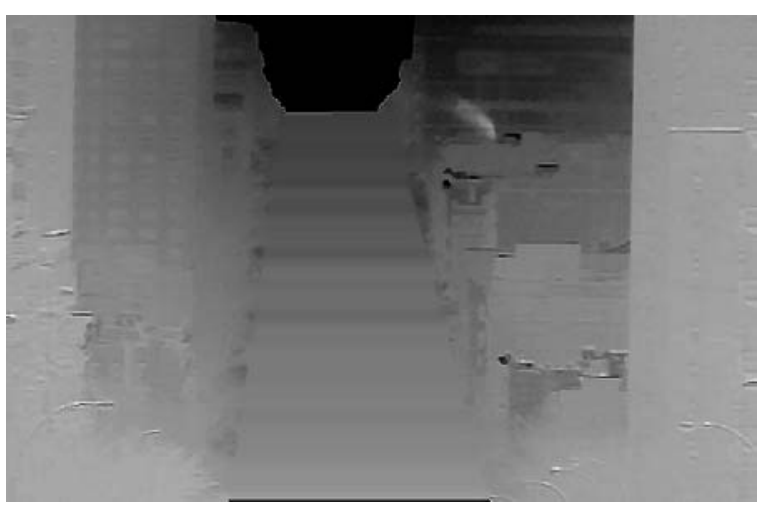

(c) Depth map computed using images in $(a)$ and $(b)$
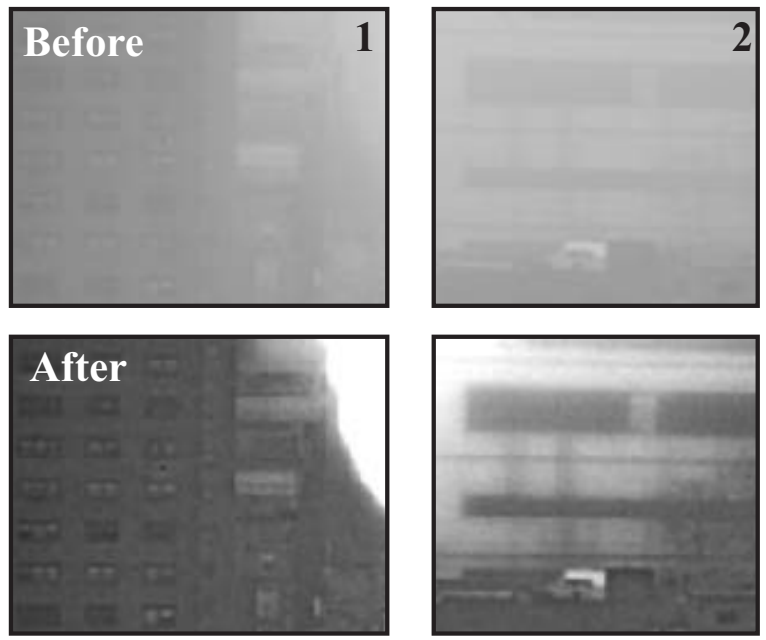

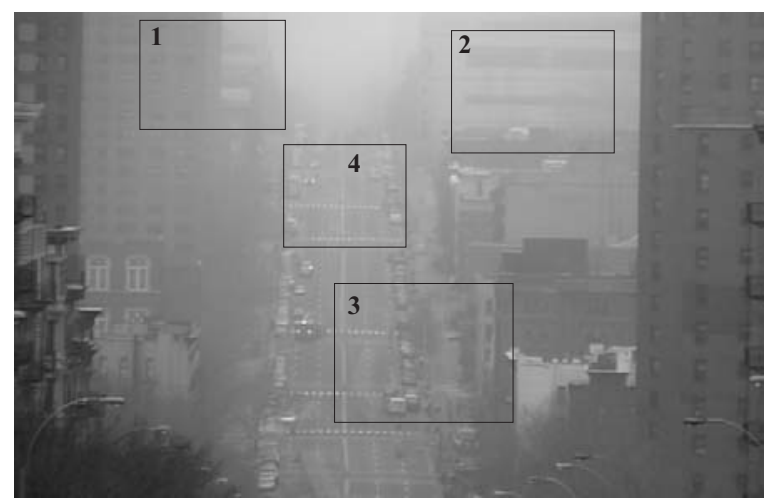

(b) Scene imaged at $5: 30 \mathrm{pm}$

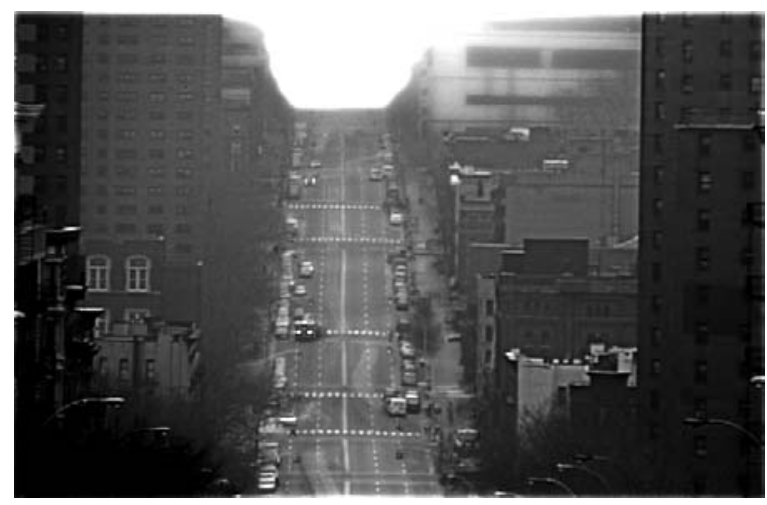

$(d)$ Contrast restored using image in $(b)$
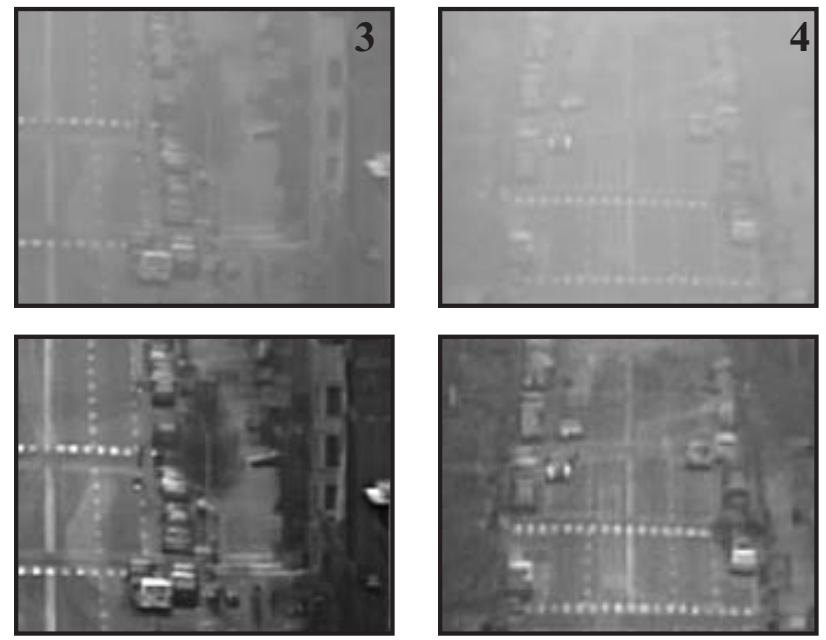

(e) Comparing contrasts of zoomed-in regions at various depths (including moving objects)

Figure 6: Experiments with videos of a traffic scene on a foggy day. $(a)-(b)$ Two short video clips were captured half an hour apart using an 8-bit video camera. 100 frames were averaged to reduce noise. Note that the vehicles on the road in the two images are different. (c) The depth map was computed for the background image using the algorithm presented in section 5 . The scaled depths of the region corresponding to the road were linearly interpolated. $(d)$ The de-fogged (contrast restored) image obtained from the video frame in $(b)$. (e) Zoomed in regions of the frame showing the significant increase in contrast at various depths of the scene. Note that different amounts of fog were removed at different depths. Also, notice the better contrast of moving objects (vehicles). Compare the contrast restored image with the histogram equalized image in Figure 7. 


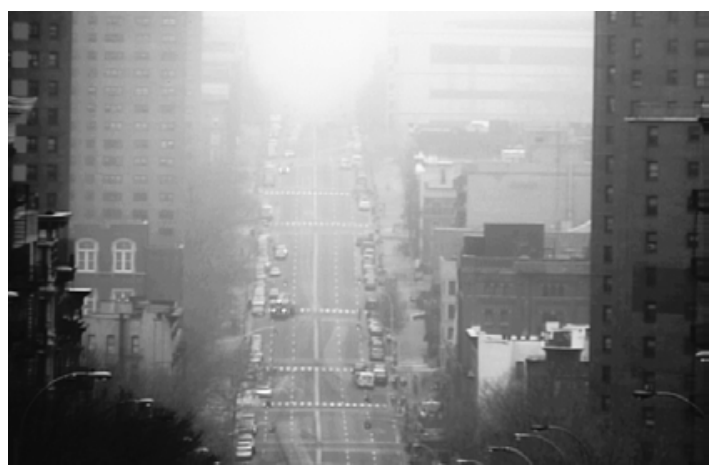

Figure 7: Artificial contrast enhancement (not contrast restoration) by histogram equalization does not remove fog completely at all depths in the scene. In this example, farther depths have poorer contrast when compared to the nearer depths.

[5] N.S. Kopeika. A System Engineering Approach to Imaging. SPIE Press, 1998.

[6] E.J. McCartney. Optics of the Atmosphere: Scattering by molecules and particles. John Wiley and Sons, 1975.

[7] W.E.K. Middleton. Vision through the Atmosphere. University of Toronto Press, 1952.

[8] M. Minnaert. The Nature of Light and Color in the Open Air. Dover Publications, Inc., 1954.

[9] S. G. Narasimhan and S. K. Nayar. Contrast restoration of weather degraded images. Columbia Univ. Tech. Report (in prep), 2001.

[10] S.G. Narasimhan and S.K. Nayar. Chromatic framework for vision in bad weather. Proceedings of the IEEE Conference on Computer Vision and Pattern Recognition, 2000.

[11] S.K. Nayar and S.G. Narasimhan. Vision in bad weather. Proceedings of the 7th International Conference on Computer Vision, 1999.

[12] J.P. Oakley and B.L. Satherley. Improving image quality in poor visibility conditions using a physical model for degradation. IEEE Trans. on Image Processing, 7, February 1998.

[13] Y.Y. Schechner, S.G. Narasimhan, and S.K. Nayar. Instant dehazing of images using polarization. Proceedings of the IEEE Conference on Computer Vision and Pattern Recognition, 2001.

[14] C. Stauffer and W.E.L. Grimson. Adaptive background mixture models for real-time tracking. Proceedings of the IEEE Conference on Computer Vision and Pattern Recognition, 1999.

[15] K. Tan and J.P. Oakley. Enhancement of color images in poor visibility conditions. International Conference on Image Processing, 2, September 2000.

[16] W.L. Wolfe and G.J. Zissis. The Infrared Handbook. Prepared for Office of Naval Research, Department of Navy, 1978.

[17] Y. Yitzhaky, I. Dror, and N.S. Kopeika. Restoration of altmospherically blurred images according to weather-predicted atmospheric modulation transfer function. Optical Engineering, 36, November 1998.

\section{Appendix}

\section{Monochrome Camera Sensing in Bad Weather}

In this section, we derive an expression for the intensity $E$, of a scene point under bad weather, recorded by a camera within a narrow wavelength band $(\lambda, \lambda+\delta \lambda)$. From (3) we write,

$$
E=\int_{\lambda}^{\lambda+\delta \lambda} s(\lambda)\left(E_{d t}(d, \lambda)+E_{a}(d, \lambda)\right) d \lambda
$$

where $s(\lambda)$ is the spectral response of the camera. We assume that the scattering coefficient $\beta$ does not change appreciably over the narrow spectral band and write,

$$
\begin{aligned}
E & =\frac{e^{-\beta d}}{d^{2}} \int_{\lambda}^{\lambda+\delta \lambda} E_{\infty}(\lambda) s(\lambda) r(\lambda) d \lambda \ldots \\
& +\left(1-e^{-\beta d}\right) \int_{\lambda}^{\lambda+\delta \lambda} E_{\infty}(\lambda) s(\lambda) d \lambda
\end{aligned}
$$

Also, we write the sky illumination spectrum as,

$$
E_{\infty}(\lambda)=I_{\infty}^{\prime} \widehat{E}_{\infty}(\lambda)
$$

where, $I^{\prime}{ }_{\infty}$ is the magnitude of the sky illumination spectrum and $\widehat{E}_{\infty}(\lambda)$ is the normalized sky illumination spectrum. Letting

$$
\begin{aligned}
g & =\int_{\lambda}^{\lambda+\delta \lambda} \widehat{E}_{\infty}(\lambda) s(\lambda) d \lambda, \\
\rho & =\frac{1}{g d^{2}} \int_{\lambda}^{\lambda+\delta \lambda} \widehat{E}_{\infty}(\lambda) s(\lambda) r(\lambda) d \lambda, \\
I_{\infty} & =I_{\infty}^{\prime} g
\end{aligned}
$$

we rewrite the final brightness at any pixel as,

$$
E=I_{\infty} \rho e^{-\beta d}+I_{\infty}\left(1-e^{-\beta d}\right),
$$

where, $I_{\infty}$ is termed as sky intensity. Note that $\rho$ is a function of normalized sky illumination spectrum, scene point reflectance and the spectral response of the camera, but not the weather condition $\beta$. The algorithm we present in the paper recovers $\rho$ for each pixel to restore scene contrast.

Let us now examine the wavelength range in which this model can be applied. By changing the limits of integration to $\left[\lambda_{1}, \lambda_{2}\right]$, and assuming the scattering coefficient to be constant over this wavelength band, we can use the same model for a black and white camera (entire visible range), or smaller color bands (R,G,B) for a color camera, or narrow band multi-spectral cameras. Thus, for removal of fog and dense haze, we can use RGB color or gray-scale cameras whereas we must use narrow spectral band cameras for the removal of many aerosols. 